\title{
Big Data in Radiation Oncology: Challenges and Opportunities
}

\author{
Jun Deng, PhD* \\ Department of Therapeutic Radiology, Yale University, New Haven, CT 06510, USA
}

Received: December 26, 2014; Accepted: December 28, 2014; Published: December 30, 2014

*Corresponding author: Jun Deng, Ph.D., Department of Therapeutic Radiology, Yale University, 15 York Street, LL508-Smilow, New Haven, CT 065103221. Tel: 203-200-2013; Fax: 203-200-2054; E-mail: jun.deng@yale.edu

Keywords: Big data; Radiation oncology; Healthcare value; Patient care; Patient safety

With coming together of a pressing demand for a value-based healthcare model to improve quality of care to cancer patients, a challenging environment for external research funding, and reduced payment for clinical services, radiation oncology community are expecting a stormy time ahead in the near future [1]. A variety of approaches has been suggested, among which full utilization of 'big data' generated in routine practices of radiation therapy is considered to be a viable solution to these imminent problems $[2,3]$.

According to Wikipedia, big data is an all-encompassing term for any collection of data sets so large and complex that it becomes difficult to process them using traditional data processing applications. The challenges include analysis, capture, curation, search, sharing, storage, transfer and visualization of these large and complex data sets [4].

In radiation oncology, big data generally consists of patient demographics stored in patient Electronic Medical Records (EMR), beam settings, plan parameters and dose volumetric information of the tumors and normal tissues generated by Treatment Planning System (TPS), 3D and 4D anatomical and functional information from diagnostic and therapeutic imaging modalities stored in Picture Archiving and Communication System (PACS), as well as genomics, proteomics and metabolomics information derived from blood and tissue specimens.

It is estimated that there are 17 million people diagnosed with cancer in 2014 worldwide, more than half of them will receive radiation therapy as part of their treatments along with surgery and chemotherapy [5]. The routine operations of modern radiotherapy treatments produce an abundance of electronically stored data on an ongoing basis, scattered across various disciplines (e.g., radiation oncology, diagnostic radiology, and neurosurgery) and data storage platforms (e.g., EMR, PACS, and database servers) in a variety of formats (e.g., DICOM, ASCII, and PDF). So far, big data generated within radiation oncology system has not been fully utilized to improve patient care, patient safety and healthcare value efficiently, due primarily to its complexity and magnitude [1-3].

Nevertheless, there is a strong urgency in utilizing big data to its full capacity without further delay. Firstly, the current feefor-service healthcare model in United States is not going to be sustainable. The United States spends about $\$ 640$ billion or $17 \%$ of its Gross Domestic Product (GDP) on health care per year, much more than any other major developed countries such as the United Kingdom, Germany and Denmark, with outcomes no better than those of its peers [6]. The continuous practice of this fee-forservice model will undoubtedly bring financial crises to federal and state governments and turmoil to health care stakeholders. It is therefore highly desirable to establish a more sustainable healthcare model based on big health care data that rewards accountability, quality, safety and value of health care provided [3]. Secondly, radiation oncology specialty spends a lot more money than other specialties and is currently facing considerable Medicare cuts from Center for Medicare and Medicaid Services (CMS) [7]. As indicated in a recent study, radiation oncology specialty is a top offender for exceeding the sustainable growth rate (SGR) target, with its excess expenditures from 2003 to 2009 hovering at $300 \%$ range as compared to 2002 [8]. To mitigate negative impact of Medicare cuts on cancer patents and radiation oncology specialty, big data can be exploited to improve treatment efficacy, optimize therapeutic ratio as well as personalize treatment options $[9,10]$. Finally, patient data generated within radiation oncology practices is currently residing in separated silos, and has so far not translated into some actionable insights into the best ways of treating patients who are the source of these data. If such big data can be assembled efficiently and analyzed systematically, great statistical power derived from these largescale population-based analyses can be leveraged to assist in clinical decision making and prediction of treatment outcomes $[9,10]$.

In fact, there has been a variety of novel applications with big data in radiation oncology recently. As indicated in this pilot study, researchers have started to use big data compiled on a large-scale prostate cancer data registry to identify best treatment strategy that allows patients to safely choose to do nothing, opt for relatively mild treatments or take drastic measures such as surgery [11]. Insights gained from this kind of population-based study would largely help radiation oncologists in clinical decision making. Another application is for enhanced patient safety where radiation physicists gain great confidence in treatment plan optimization, quality assurance and treatment delivery, based 
on a standardized database derived from big data contributed by multiple institutions $[9,10]$. Furthermore, radiobiologists can gain insights into treatment response modeling and outcome prediction with the help of big data. For instance, genetic profiling based on collaborative efforts of multiple institutions could help guide cancer patients and their doctors to select the best treatment approach or radiotherapy dose for each patient [12].

The era of big data in radiation oncology has arrived, which presents many challenges as well as opportunities for not only care providers in cancer radiotherapy but also stakeholders in health care and government. With effective and appropriate tools, big data in radiation oncology can be analyzed and utilized to assist in decision making for clinicians, personalize treatment strategy for individual patient, and improve patient safety and treatment efficacy, leading to a more cost-effective healthcare model with better outcomes and quality of care in the long run.

\section{References}

1. Pearson D. The Near Future Comes into Clear(er) Focus. Imagingbiz. [Dated 2014 Dec 8] Available from: http://www.imagingbiz.com/ sponsored/1065/near-future-comes-clearer-focus?nopaging=1.

2. Fratt L. The New Economics of Radiation Oncology. Health Imaging [Dated 2013 May 6] Available from: http://www.healthimaging. com/topics/healthcare-economics/new-economics-radiationoncology?nopaging=1.

3. Schneeweiss S, Learning from Big Health Care Data. N Engl J Med. 2014; 370: 2161-3.
4. Wikipedia. Big data. Availavble form: http://en.wikipedia.org/wiki/ Big_data.

5. Ferlay J, Soerjomataram I, Ervik M, et al. GLOBOCAN 2012 v1.0, Cancer Incidence and Mortality Worldwide: IARC CancerBase No. 11 [Internet]. Lyon, France: International Agency for Research on Cancer; 2013.

6. Fuchs VR, Milstein A. The $\$ 640$ billion question--why does costeffective care diffuse so slowly? N Engl J Med. 2011; 364(21):1985-7.

7. Neumann PJ, Tunis SR. Medicare and Medical Technology - The Growing Demand for Relevant Outcomes. N Engl J Med. 2010; 362(5):377-9.

8. Alhassani A, Chandra A, Chernew ME. The Sources of the SGR "Hole". N Engl J Med. 2012; 366:289-291.

9. Moore KL, Kagadis GC, McNutt TR, Moiseenko V, Mutic S. Vision 20/20: Automation and advanced computing in clinical radiation oncology. Med Phys. 2014(1); 010901.

10.Skripcak T, Belka C, Bosch W, Brink C, Brunner T, Budach V, et al. Creating a data exchange strategy for radiotherapy research: Towards federated databases and anonymised public datasets. Radiotherapy and Oncology. 2014; 113: 303-9.

11. Mayhood K. Using big data to identify prostate cancers and best treatments. AAAS. Available form: http://www.eurekalert.org/pub_ releases/2013-05/cwru-ubd052313.php.

12. Rosenstein BS, West CM, Bentzen SM, Alsner J, Andreassen CN, Azria D, et al. Radiogenomics: Radiobiology Enters the Era of Big Data and Team Science. Int J Radiat Oncol Biol Phys. 2014; 89:709-13. 